\title{
Rapamycin promotes osteogenesis under inflammatory conditions
}

\author{
XING LI $^{1 *}$, BEI CHANG ${ }^{1 *}$, BANCHAO WANG ${ }^{1}$, WENHUAN BU ${ }^{1}$, LIANG ZHAO ${ }^{1}$, JIE LIU ${ }^{1}$, LIN MENG ${ }^{1}$, \\ LU WANG $^{1}$, YING XIN ${ }^{1}$, DANDAN WANG ${ }^{1}$, QI TANG ${ }^{1}$, CHANGYU ZHENG $^{2}$ and HONGCHEN SUN ${ }^{1}$ \\ ${ }^{1}$ Department of Oral Pathology, School and Hospital of Stomatology, Jilin University, Changchun, Jilin 130021, P.R. China; \\ ${ }^{2}$ Molecular Physiology and Therapeutics Branch, National Institute of Dental and Craniofacial Research, \\ National Institutes of Health, Bethesda, MD 20892, USA
}

Received April 26, 2017; Accepted September 19, 2017

DOI: $10.3892 / \mathrm{mmr} .2017 .7693$

\begin{abstract}
Chronic periodontitis, a common oral disease, usually results in irreversible bone resorption. Bone regeneration is a complex process between bone-forming activity of osteoblasts and bone-resorbing activity of osteoclasts, and still remains a challenge for physicians clinically. A previous study demonstrated that the mechanistic target of rapamycin signaling pathway is involved in osteogenic differentiation of mesenchymal stromal cells. Herein, whether rapamycin could be used to induce osteogenic differentiation of primary bone marrow-derived mesenchymal stem cells (BMSCs) in vitro and promote new bone formation in vivo were evaluated. The results demonstrated that rapamycin alone was not enough to fully induce osteoblast differentiation in vitro and enhanced bone regeneration in vivo. Interestingly, rapamycin in rapamycin plus lipopolysaccharide (LPS)-treated BMSCs significantly increased the gene expression levels of Sp7 transcription factor, runt related transcription factor 2, alkaline phosphatase (ALP) and collagen I (Col I), ALP activity, and calcium nodule at different time points in vitro, indicating that osteoblast differentiation occurs by rapamycin when BMSCs are exposed to LPS simultaneously. It was also demonstrated that rapamycin in rapamycin plus LPS-treated rats promoted bone regeneration in vivo. These results suggest that rapamycin may influence osteoblast differentiation and new bone formation after LPS induces an inflammatory environment. Rapamycin may be used to treat periodontitis associated with bone loss in future clinical practice.
\end{abstract}

Correspondence to: Dr Hongchen Sun, Department of Oral Pathology, School and Hospital of Stomatology, Jilin University, 1500 Qinghua Road, Changchun, Jilin 130021, P.R. China

E-mail: hcsun@mail.jlu.edu.cn

${ }^{*}$ Contributed equally

Key words: rapamycin, periodontitis, LPS, osteoblast, osteogenesis, bone regeneration

\section{Introduction}

Bone is a tissue that is constantly being remodeled during a human's or animal's life span through the activity or interaction of bone generating osteoblasts and bone resorbing osteoclasts (1). The balance between bone resorption and bone formation is essential for maintaining bone homeostasis. Many molecules are involved in communication between osteoblasts and osteoclasts during bone remodeling, such as receptor activator of nuclear factor $\kappa B$ ligand (RANKL)/receptor activator of nuclear factor $\kappa B$ (RANK), ephrinB2/EphB4, TGF- $\beta$, and so on (2). Recently, many studies have demonstrated that the immune system is also involved intimately in the regulation of bone regeneration (3). Extensive evidence suggests that the immune system exerts powerful effects on bone cells, particularly in chronic disease pathologies such as rheumatoid arthritis, periodontal disease, and tumors $(4,5)$.

As a common disease identified in patients visiting oral clinics, the chronic inflammatory state in periodontitis triggers bone erosion through the increased stimulation of osteoclast formation and activity, decreasing the chance for preservation of permanent teeth and the survival of implants (4). Lipopolysaccharide (LPS), an important virulence factor of periodontitis, stimulates the accumulation of monocytes, releases many inflammatory factors, and is the cause of maturation of osteoclasts. Furthermore, T lymphocytes play an important role in regulation of bone mass in inflammatory diseases. T cells can synthesize RANKL and induce osteoclast formation and activity in vitro (4-6). Considering the crucial effect of immune factors on the development of periodontitis, it raises an interesting question of whether or not clinicians can use immunosuppressive medicine to block bone resorption.

Rapamycin is a common immunosuppressive drug that has multiple functions such as radiation protection and prevention of aging, inhibition of tumor growth, regulating endothelial cell growth, inhibition of adipogenic differentiation of bone marrow-derived mesenchymal stem cells (BMSCs), among others (7-11). Rapamycin also plays important roles in bone homeostasis, which regulates bone formation through the $\mathrm{mTOR} / \mathrm{S} 6 \mathrm{~K}$ and nuclear factor- $\mathrm{\kappa B}(\mathrm{NF}-\mathrm{\kappa B})$ signaling pathway (12). mTOR is an evolutionally conserved serine/threonine protein kinase involved in many cellular processes such as survival and proliferation (13). Rapamycin is a specific inhibitor of mTOR, and can downregulate expression of 
RANKL, M-CSF, and tumor necrosis factor $\alpha(\mathrm{TNF} \alpha)$, and exerts suppressive effects on proliferation and differentiation of osteoclasts (14). More importantly, rapamycin can inhibit the release of inflammatory factors $(15,16)$, which further reduces differentiation of osteoclasts. The role of rapamycin in osteogenesis, however, still remains controversial because conflicting studies have reported both negative (17-19) and positive $(20,21)$ effects on osteogenesis. These differences may depend on cell type and experimental conditions.

Periodontitis is a chronic inflammatory disease resulting in bone loss, and rapamycin plays a role as an anti-inflammatory and possibly has an effect on bone formation. Therefore, we were interested in evaluating the effects of rapamycin on osteoblast differentiation of BMSCs in vitro, and bone regeneration in our animal model in vivo, with or without LPS treatment, in order to understand if rapamycin can be used to treat periodontitis.

\section{Materials and methods}

Cell lines and cell cultures. All animals used for this study were approved by the Jilin University (Changchun, China) Animal Care and Use Committee. In this study, BMSCs were obtained from the femur of Wistar rats. BMSCs were cultured/expanded in low-glucose DMEM (Thermo Fisher Scientific, Inc., Grand Island, NY, USA)) with $10 \%$ fetal bovine serum, $100 \mathrm{U} / \mathrm{ml}$ penicillin, and $100 \mathrm{mg} / \mathrm{ml}$ streptomycin. To perform osteoblast differentiation, BMSCs were cultured in low-glucose DMEM (Thermo Fisher Scientific, Inc.) supplemented with $10^{-8} \mathrm{~mol} / 1$ dexamethasone (Sigma-Aldrich, St. Louis, MO, USA), $50 \mathrm{mg} / 1$ of ascorbic acid (Sigma-Aldrich), and $10 \mathrm{mM} \beta$-glycerol phosphate sodium (Merck, Kenilworth, NJ, USA) known as osteogenic medium with $10 \%$ fetal bovine serum, $100 \mathrm{U} / \mathrm{ml}$ penicillin, and $100 \mathrm{mg} / \mathrm{ml}$ streptomycin. Cells were incubated at $37^{\circ} \mathrm{C}$ in a humidified $5 \% \mathrm{CO}_{2}$ incubator.

Alkaline phosphatase (ALP) and alizarin red assays. BMSCs cells were seeded/cultured in a 6 -well plate at $1 \times 10^{4}$ cells/well with low-glucose DMEM (Thermo Fisher Scientific, Inc.) for $24 \mathrm{~h}$. Then, plates divided into four groups, control phosphate-buffered saline (PBS)], LPS at $1 \mu \mathrm{g} / \mathrm{ml}$ (Sigma-Aldrich), rapamycin at $20 \mathrm{ng} / \mathrm{ml}$ (Rapa, Sigma-Aldrich), and LPS at $1 \mu \mathrm{g} / \mathrm{ml}$ plus rapamycin at $20 \mathrm{ng} / \mathrm{ml}$ (LPS + Rapa), and continue to culture with osteogenic medium. The osteogenic medium with or without LPS or rapamycin was replaced every 3 days. On days 3, 7, and 14, ALP activity assay was performed with ALP substrate (Sigma-Aldrich), and optical density (OD) was measured at $520 \mathrm{~nm}$ with a spectrometer according to the manufacturer's instructions. On days 14,21 , and 28 cells from the same experiments were fixed with $95 \%$ ethanol and stained with $2 \%$ alizarin red (Sigma-Aldrich). The stained cells were then eluted with $10 \%$ cetylpyridinium chloride. The absorbance was measured at $550 \mathrm{~nm}$ with a spectrometer.

Reverse transcription quantitative polyermerase chain reaction $(R T-q P C R)$. BMSCs were seeded in a 6-well plate at $1 \times 10^{4}$ cells/well and cultured with osteogenic medium for $24 \mathrm{~h}$. Then, plates divided into four groups, control (PBS), LPS at $1 \mu \mathrm{g} / \mathrm{ml}$ (Sigma-Aldrich), rapamycin at $20 \mathrm{ng} / \mathrm{ml}$ (Rapa, Sigma-Aldrich), and LPS at $1 \mu \mathrm{g} / \mathrm{ml}$ plus Rapa at $20 \mathrm{ng} / \mathrm{ml}$
(LPS + Rapa). On days 3, 7, and 14, total RNA was extracted with EASYspin (Takara, Dalian, China), and reverse transcription was carried out using $1 \mu \mathrm{g}$ total RNA with PrimerScript RT Reagent kit (Takara), The qPCRs were performed using SYBR Premix Ex Taq TM (Takara). Sequences of primers are listed in Table I. The $\beta$-actin was used as the internal control. $\mathrm{TNF} \alpha$ and interleukin (IL)- $1 \beta$ are important mediators of the inflammatory response. Runt related transcription factor 2 (Runx2) is a necessary protein in the early and middle stages of osteogenic differentiation (22) and acts as an essential bone specific transcription factor (23). Collagen I (Col I) is an important protein for bone formation and repair (24). ALP is a very common bone formation marker (25). Sp7 is a transcription factor and indicator for osteogenic differentiation (26) and is involved in osteoblast differentiation (27).

Animal model. A classical animal model, alveolar bone regeneration model was used to evaluate effects of rapamycin on bone regeneration in vivo (28). To successfully create this model, Wistar rats were anesthetized with ketamine $(60 \mathrm{mg} / \mathrm{kg})$ and xylazine $(8 \mathrm{mg} / \mathrm{kg})$ intramuscularly, and the crown of the left mandibular incisor was repeatedly cut at the gingival level with a high-speed turbine drill on days 8,5 , and 2 prior to the mandibular incisor being fully extracted on day 0 . The mandibular incisor of rodents keep erupting over their life span. Once the crown of the mandibular incisor is removed, the tooth will erupt more quickly, resulting in edema of the periodontal ligaments (28), which can facilitate the extraction of the mandibular incisor.

In vivo animal experiment. All animals used in this study were approved by the Jilin University Animal Care and Use Committee. Eighty male Wistar rats $(210 \pm 10 \mathrm{~g}$, and 7 to 8 weeks old) were randomly divided into three groups on day 0 of alveolar bone regeneration model: 20 rats for the control group administered with $10 \mu \mathrm{l}$ of PBS, 20 rats for the Rapa group administered with $10 \mu \mathrm{l}$ of rapamycin at $1 \mu \mathrm{g} / \mu \mathrm{l}$ into the tooth socket, 20 rats for the LPS group administered with $10 \mu \mathrm{l}$ of LPS at $5 \mu \mathrm{g} / \mu \mathrm{l}$ into the tooth socket, and 20 rats for the LPS + Rapa group administered with $10 \mu \mathrm{l}$ of LPS at $5 \mu \mathrm{g} / \mu \mathrm{l}$ with rapamycin at $1 \mu \mathrm{g} / \mu \mathrm{l}$ into the tooth socket. Defects were closed by periodontal dressing. Rats were anesthetized and euthanized by heart perfused fixation with $4 \%$ paraformaldehyde solution after 30 and 60 days post-treatment. Mandible samples were examined with micro-computed tomography (micro-CT) for bone mass. Tooth extraction sockets from different treated groups were immersed in 10\% EDTA for decalcification for two months, 10\% EDTA was refreshed every two days. After decalcification, samples were embedded in paraffin and tissues were sectioned at $4 \mu \mathrm{m}$ for hematoxylin and eosin (H\&E) staining to evaluate pathological changes.

Micro-CT. The left mandible was placed in a tube and scanned over the entire mandible using a micro-CT system ( $\mu$ CT50; Scanco Medical, Bassersdorf, Switzerland). The same condition was used to scan all samples. Three rats from each group were scanned by micro-CT. A series of two-dimensional image data was collected to reconstruct three-dimensional images. Regions of interest (ROI) were confined to the anterior area of the molar region of the mandible near the inferior 
Table I. Quantitative polymerase chain reaction primers used in this study.

\begin{tabular}{lllr}
\hline Gene & \multicolumn{1}{c}{ Forward primer $\left(5^{\prime}-3^{\prime}\right)$} & \multicolumn{1}{c}{ Reverse orimer (5'-3') } & Size (base pair, bp) \\
\hline ALP & CTCAACACCAATGTAGCCAAGAATG & GGCAGCGGTT ACTGTGGAGA & 75 \\
Runx2 & GCACAAACATGGCCAGATTCA & AAGCCATGGTGCCCGTTAG & 126 \\
Sp7 & GCACAAACATGGCCAGATTCA & AGAAATCTACGAGCAAGGTCTCCAC & 129 \\
Col I & GACATGTTCAGCTTTGTGGACCTC & GGGACCCTTAGGCCATTGTGTA & 119
\end{tabular}

ALP, alkaline phosphatase; Runx2, runt related transcription factor 2; Sp7, Sp7 transcription factor; Col I, collagen I.

border. This ROI was further analyzed using a fixed global threshold of $22 \%$ (220 on a grayscale of $0-1,000)$. Trabecular bone volume fraction (BV/TV), trabecular thickness (Tb.Th), trabecular number (Tb.N), trabecular separation (Tb.Sp), bone mineral density (BMD), and tissue mineral density (TMD) were analyzed using the manufacturer's evaluation software.

Statistical analysis. Results were presented as mean \pm standard deviation (SD). Statistical analysis was performed using the one-way ANOVA and multiple comparison method. A difference with a P-value of $<0.05$ was deemed as statistically significant. All in vitro experiments were repeated three times; in vivo experiments were repeated twice.

\section{Results}

Effects of LPS on inflammatory cytokines and mTOR. To confirm the LPS used here has the expected biological effects, the gene expressions of TNF $\alpha$, IL- $1 \beta$ and mTOR were measured after BMSCs were cultured with LPS at $1 \mu \mathrm{g} / \mathrm{ml}$ (Sigma-Aldrich) for $24 \mathrm{~h}$. Fig. 1 showed that the LPS dramatically induced the gene expressions of TNF $\alpha$, IL-1 $\beta$ and mTOR compared to the control group. These indicate that the LPS has expected biological effects.

Effects of rapamycin on osteoblast-related gene expression. To evaluate if rapamycin can induce osteogenic differentiation at the gene expression level, four marker genes, Runx2, Sp7, Col I, and ALP for osteogenic differentiation, were measured after BMSCs were treated with rapamycin, LPS, or rapamycin plus LPS by RT-qPCR on days 3, 7, and 14. Rapamycin alone significantly decreased gene expression of Runx2, but not Sp7, Col I, or ALP compared to the control group on day 3 (Fig. 2A). LPS alone decreased gene expressions of Runx2 and Col I, but not ALP and Sp7 compared to the control group on day 3 (Fig. 2A). LPS plus rapamycin also decreased gene expressions of Runx2 and Col I, but increased ALP and Sp7 compared to the control group on day 3 (Fig. 2A). On day 7, rapamycin alone only increased Col I gene expression while LPS alone increased gene expressions of Runx2, Col I, and ALP compared to the control (Fig. 2B). Interestingly, LPS plus rapamycin significantly increased gene expressions of Runx2, Col I, ALP, and Sp7 compared to the control on day 7 (Fig. 2B). Rapamycin downregulated gene expressions of Runx2, Col I, and Sp7 while LPS decreased gene expressions of Runx 2 and Sp7 compared to the control on day 14 (Fig. 2C). Both LPS and LPS plus rapamycin increased gene expressions of Col I and ALP compared to the
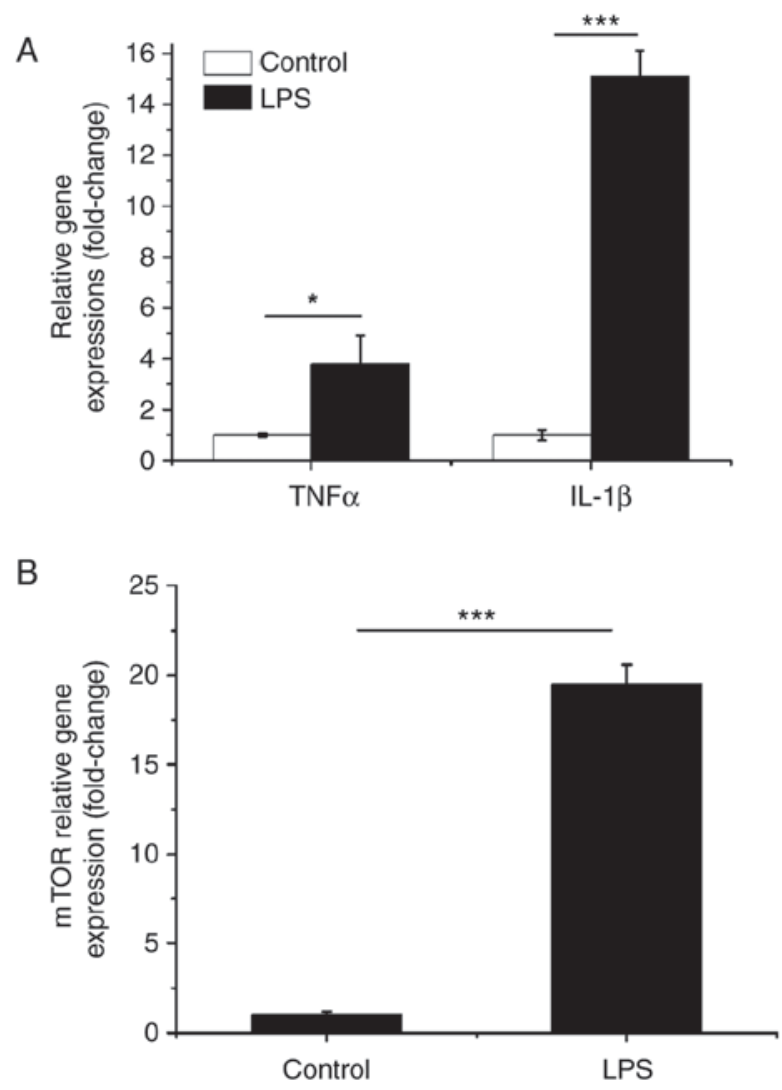

Figure 1. Gene expressions of TNF $\alpha$, IL-1 $\beta$ and mTOR after BMSCs treated with LPS $(1 \mu \mathrm{g} / \mathrm{ml})$ for $24 \mathrm{~h}$. (A) Gene expressions of TNF $\alpha$ and IL-1 $1 \beta$. (B) Gene expression of $\mathrm{mTOR}$. Data are represented as means \pm SD from three experiments. ${ }^{*} \mathrm{P}<0.05,{ }^{* * * *} \mathrm{P}<0.001$. TNF $\alpha$, tumor necrosis factor $\alpha$; IL, interleukin; BMSCs, bone marrow-derived mesenchymal stem cells; LPS, lipopolysaccharide; SD, standard deviation.

control on day 14 (Fig. 2C). These data indicate that rapamycin plus LPS can induce osteoblast differentiation.

Rapamycin plus LPS increased ALP activity. Next, we directly measured ALP activity in BMSC cultures to assess osteoblast differentiation. Interestingly, only rapamycin plus LPS enhanced ALP activity compared to the control, rapamycin and LPS groups on days 3, 7, and 14 (Fig. 3). These data demonstrate that the rapamycin alone is not enough to affect osteoblast differentiation while the rapamycin plus LPS can induce osteoblast differentiation in vitro.

Effects of rapamycin on formation of calcium nodule. Alizarin red staining is used to identify calcium in tissue section or 

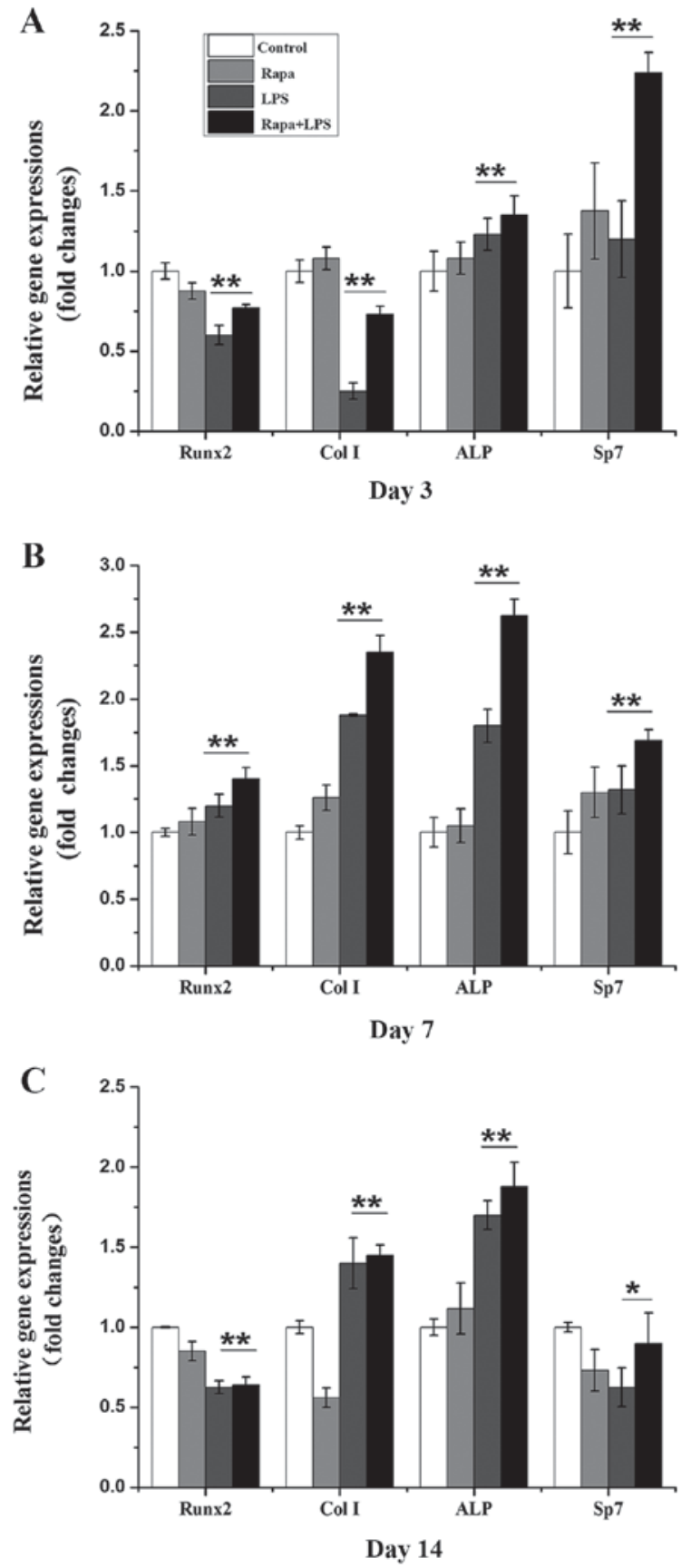

Figure 2. Gene expressions of Runx2, Col I, ALP, and Sp7 after 3, 7, and 14 days post-treatment. (A) Gene expressions on day 3. (B) Gene expressions on day 7. (C) Gene expressions on day 14. Data are represented as means \pm SD from three experiments. ${ }^{*} \mathrm{P}<0.05,{ }^{* *} \mathrm{P}<0.01$. Runx 2 , runt related transcription factor 2; Col I, collagen I; ALP, alkaline phosphatase; Sp7, Sp7 transcription factor; SD, standard deviation; Rapa, rapamycin; LPS, lipopolysaccharide.

cell culture which can indicate osteogenesis differentiation. The data clearly showed that only LPS plus rapamycin had significantly more calcium nodule compared to any other groups on days 14, 21, and 28 (Fig. 4). These data further suggest that the rapamycin plus LPS can promote osteoblast differentiation in vitro.

Effect of rapamycin on bone formation in vivo. To further evaluate the effect of rapamycin on bone formation, we performed in vivo experiments using the alveolar bone regeneration model. Data from BV/TV, BMD, TMD, Tb.N,

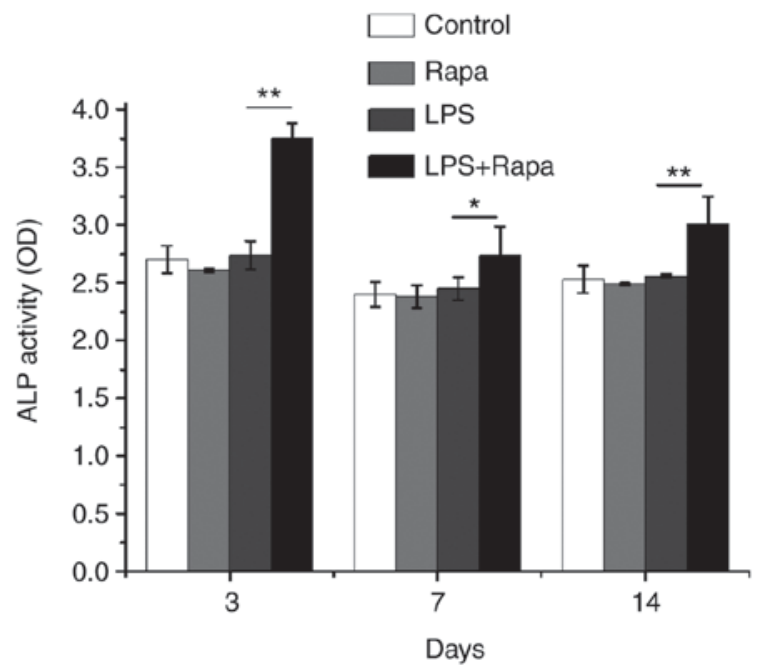

Figure 3. ALP activity assay after 3, 7, and 14 days post-treatment. Data are represented as means \pm SD from three experiments. ${ }^{*} \mathrm{P}<0.05,{ }^{* * *} \mathrm{P}<0.01$. ALP, alkaline phosphatase; SD, standard deviation; OD, optical density; Rapa, rapamycin; LPS, lipopolysaccharide.

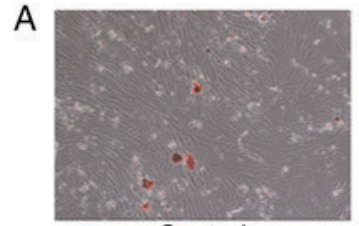

Control

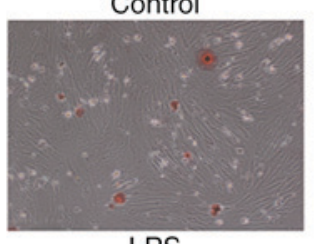

LPS

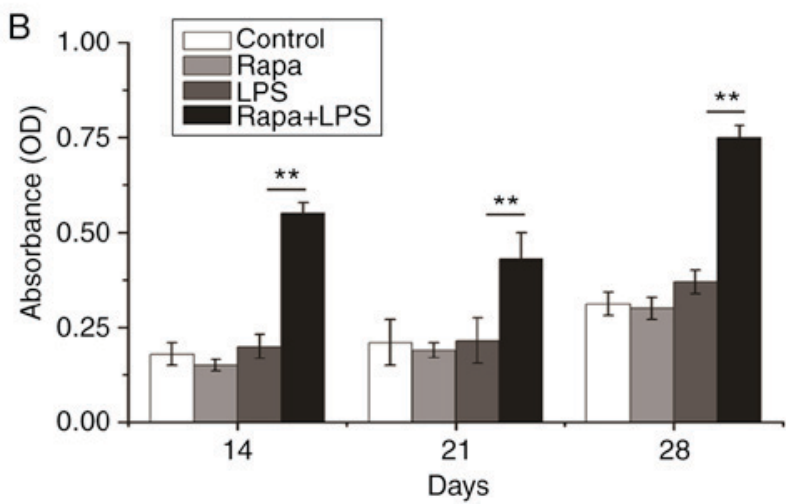

Figure 4. Calcium nodule examination by alizarin red staining after 14, 21 , and 28 days post-treatment. (A) Observation under microscope. (B) Bar graph calculated from OD measurement. Data are represented as means \pm SD from three experiments. ${ }^{* *} \mathrm{P}<0.01$. OD, optical density; $\mathrm{SD}$, standard deviation; Rapa, rapamycin; LPS, lipopolysaccharide.

and $\mathrm{Tb} . \mathrm{Th}$ showed that rapamycin plus LPS significantly increased bone formation compared to PBS control and rapamycin control (data not shown here) on weeks 4 and 8 while LPS alone only significantly increased Tb.Th, and both LPS and rapamycin plus LPS decreased Tb.Sp (Fig. 5). H\&E staining from Fig. 6 clearly demonstrated that the 

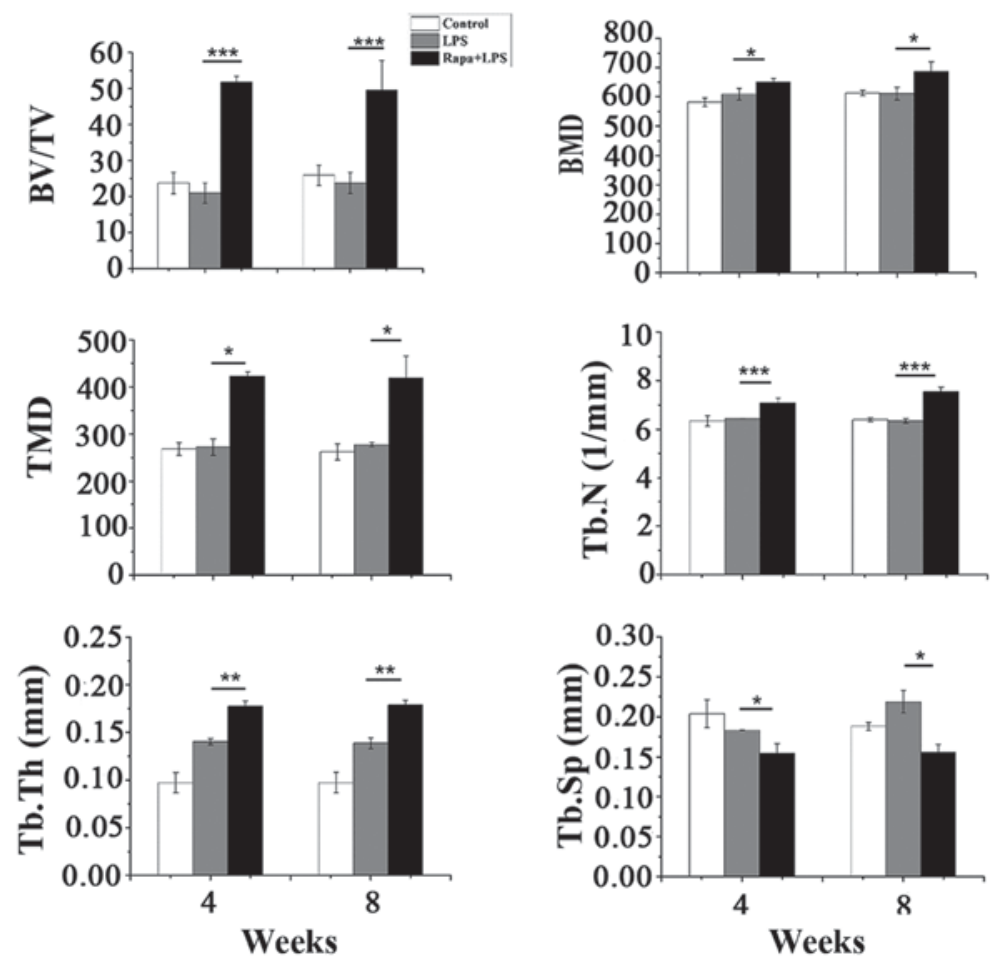

Figure 5. Measurements of BV/TV, BMD, TMD, Tb.N, Tb.Th, and Tb.Sp of mandible trabecular bone after 4 and 8 weeks post-treatment. Data are represented as means \pm SD from 20 rats. ${ }^{*} \mathrm{P}<0.05,{ }^{* *} \mathrm{P}<0.01,{ }^{* * *} \mathrm{P}<0.001$. BV/TV, trabecular bone volume fraction; BMD, bone mineral density; TMD, tissue mineral density; Tb.N, trabecular number; Tb.Th, trabecular thickness; Tb.Sp, trabecular separation; SD, standard deviation; Rapa, rapamycin; LPS, lipopolysaccharide.

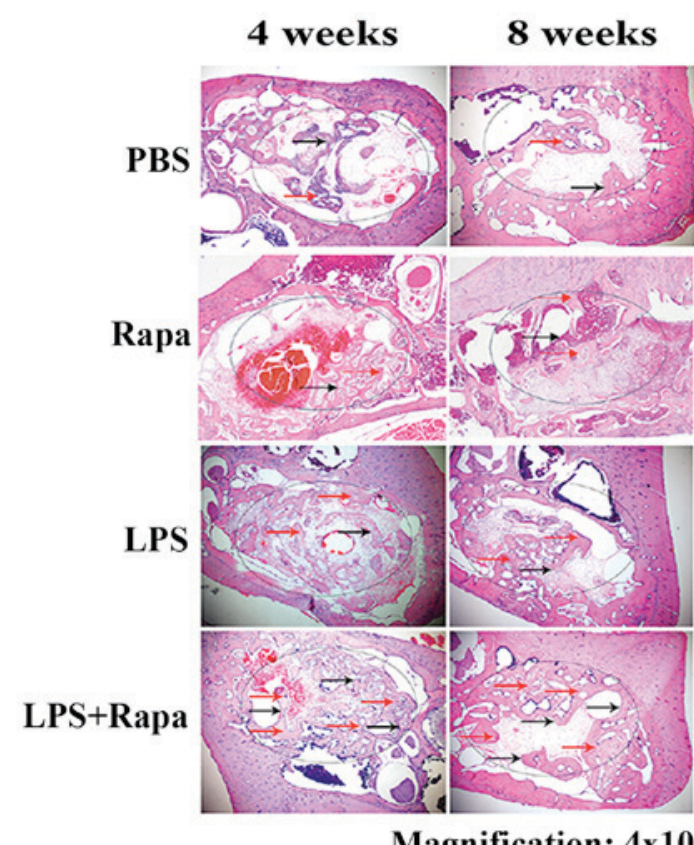

Magnification: 4x10

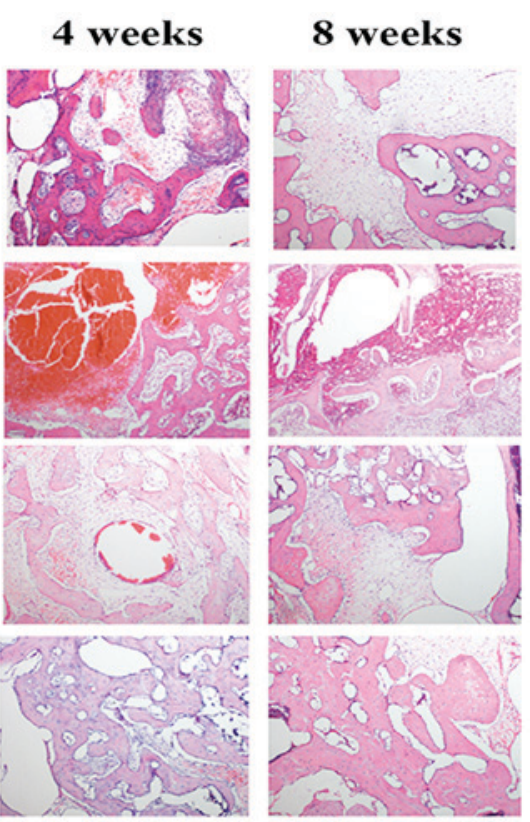

Magnification: 10x10

Figure 6. Data of H\&E staining of mandible trabecular bone after 4 and 8 weeks post-treatment. Black arrow indicates osteoblast. Red arrow indicates new bone formation. H\&E, hematoxylin and eosin; PBS, phosphate-buffered saline; Rapa, rapamycin; LPS, lipopolysaccharide.

rapamycin plus LPS-treated group had much more new bone formation with scattered osteoblasts compared to control, Rapa and LPS groups on weeks 4 and 8 . At 8 weeks, bone deposition continued to increase, and the new bone showed bigger and higher density of bone trabecula in rapamycin plus LPS-treated group (Fig. 6). H\&E staining data were consistent with the micro-CT data. These data suggest that rapamycin affects bone homeostasis by stimulating osteoblast activity under LPS-induced inflammatory condition.

\section{Discussion}

Osteogenesis is one of two major processes, a process of new bone formation by osteoblast in bone remodeling. Bone 
resorption is another process by osteoclast in bone remodeling. It is important to maintain the balance between bone formation and bone resorption. Complex molecules and signaling pathways are involved in these processes. Rapamycin is a common immunosuppressant widely used to retain a renal allograft. Recently, it was recognized that mammalian target of rapamycin complex 2 (mTORC2) signaling pathway is involved in osteogenesis processes, and that rapamycin can also affect bone homeostasis under certain conditions, but this is still unclear and remains controversial (29). For example, it showed that osteoblast-targeted inhibition of PPARc activated mTOR signaling pathway resulting in osteoblast differentiation (30) while Wang et al demonstrated that the rapamycin inhibited mTOR signaling pathway leading to BMSCs differentiation/proliferation to osteoblasts (31). The study herein indicates that rapamycin can induce osteoblast differentiation and lead to new bone formation under LPS-induced inflammatory conditions.

Bacterial endotoxin LPS is a potent stimuli for monocytes, which produce pro-inflammatory cytokines such as TNFa, IL-1, and IL-6 to play an important role in pathogenesis bacterial sepsis (32-34). LPS can also activate NF- $\mathrm{BB}$ (35). Interestingly, cytokines of TNF $\alpha$, IL-1, and IL- 6 can play critical roles in osteoclast formation resulting in osteoclastic bone loss $(36,37)$. These cytokines can induce production of RANKL $(38,39)$, decrease production of osteoprotegerin (OPG) (40), upregulate RANK on osteoclast precursors, and increase RANKL to affect RANK/RANKL/OPG signaling pathway (41). LPS can also interact with toll-like receptor (TLR)-2 and/or TLR-4, resulting in RANKL expression or release increase and manipulation of RANK/RANKL/OPG signal pathway (41).

It is known that rapamycin possesses potent anti-proliferative and anti-angiogenic properties (42). Previous studies show that rapamycin downregulates expressions of RANKL, $\mathrm{M}-\mathrm{CSF}$, and TNF $\alpha$ to suppress proliferation and differentiation of osteoclast, and also block cytokine release to further inhibit differentiation of osteoclast $(15,16)$. These data indicate that rapamycin potentially reduces bone resorption and contributes to a bone-protective effect or new bone formation through anti-inflammatory influence $(20,21)$. Rapamycin could partly activate osteocyte autophagy and reduce age-related bone loss in trabecular bone of old male rats (43). Indeed, it has been reported that rapamycin could also inhibit osteoblast proliferation and differentiation in MC3T3-E1 cells and primary mouse bone marrow stromal cell (17). Therefore, there is uncertain effect of rapamycin on bone formation.

Our data demonstrate that rapamycin alone did not influence gene expression of Runx2, Col I, ALP, and Sp7 compared to the control group on days 3,7 , and 14, except that it slightly decreased Runx 2 on days 3 and 14 (Fig. 2). Rapamycin alone also could not increase ALP enzyme activity on days 3, 7, and 14 (Fig. 3). LPS decreased gene expression of Runx 2 and Col I compared to the control group on days 3 and 14, and Sp7 on day 14 (Fig. 1), and also had no effect on ALP enzyme activity on days 3, 7 and 14 (Fig. 3). Interestingly, rapamycin from the rapamycin plus LPS group could either enhance or bring back gene expression of Runx2, Col I, ALP, and Sp7 compared to LPS alone on all three days in which LPS induced an increase or decrease (Fig. 2), and also increased ALP enzyme activity (Fig. 3). Data from calcium nodule assay showed that only rapamycin plus LPS-treated BMSCs significantly increased the number of calcium nodules (Fig. 4). These data clearly indicate that rapamycin alone cannot fully induce BMSC differentiation to become functional osteoblasts in vitro; it only occurred when rapamycin plus LPS was used in vitro. Recently published study, however, demonstrates that rapamycin can induce their BMSCs differentiation to the osteoblast in vitro (31). Compared both culture systems, we recognized that the difference could cause by differences of culture contents and species of rat.

In our in vivo animal model (although we knew that this model was not perfect model), we delivered rapamycin locally to the extraction defect area. Micro-CT data show that bone tissue volume markedly increased in the defect area and that density of alveolar bone significantly increased in the LPS plus rapamycin-treated group (Fig. 5). H\&E staining revealed that rapamycin from the LPS plus rapamycin-treated group markedly increased new bone formation with scattered osteoblasts in the defect area (Fig. 6). Therefore, our data suggest that LPS is a precondition for rapamycin induction of osteoblast differentiation and new bone formation.

Results from our cell culture model and animal model clearly indicate that rapamycin alone is not enough to fully promote osteoblast differentiation in vitro and in vivo. However, when LPS creates an inflammatory condition resulting in cytokine production and leads RANK/RANKL/OPG signaling pathway to activate osteoclasts, then rapamycin may block the inflammation and turn around the RANK/RANKL/OPG signaling pathway to the osteoblast differentiation and new bone formation in vitro and in vivo.

Periodontitis is a chronic inflammatory disease with dense inflammatory cell infiltrates. Porphyromonas gingivalis is the major bacterial cause of periodontitis. The LPS produced by Porphyromonas gingivalis can induce cytokine production and increase soluble RANKL release to affect RANK/RANKL/OPG signaling pathway resulting in bone loss (41). Therefore, our results suggest that rapamycin can be used to treat periodontitis in order to block bone resorption and promote bone regeneration.

\section{Acknowledgements}

We would like to thank Cindy Clark, NIH Library Editing Service, for reviewing and editing the manuscript. We thank Guibin Zhu, Medical Laboratory, for her excellent technical assistance. This study was supported by grants from the National Key Research and Development Program of China (2016YFC1102800), the National Natural Science Foundation of China (81320108011, 81600879, 30830108, 81500820 and 81400488 ) and the JLU Science and Technology Innovative Research Team (2017TD-11).

\section{References}

1. Hattner R, Epker BN and Frost HM: Suggested sequential mode of control of changes in cell behaviour in adult bone remodelling. Nature 206: 489-490, 1965.

2. Li C, Shi C, Kim J, Chen Y, Ni S, Jiang L, Zheng C, Li D, Hou J, Taichman RS and Sun H: Erythropoietin promotes bone formation through EphrinB2/EphB4 signaling. J Dent Res 94: $455-463,2015$. 
3. Takayanagi H: Osteoimmunology: Shared mechanisms and crosstalk between the immune and bone systems. Nat Rev Immunol 7: 292-304, 2007.

4. Taubman MA and Kawai T: Involvement of T-lymphocytes in periodontal disease and in direct and indirect induction of bone resorption. Crit Rev Oral Biol Med 12: 125-135, 2001.

5. Dequeker J, Maenaut K, Verwilghen J and Westhovens R: Osteoporosis in rheumatoid arthritis. Clin Exp Rheumatol 12 (13 Suppl): S21-S26, 1995.

6. Kotake S, Udagawa N, Hakoda M, Mogi M, Yano K, Tsuda E, Takahashi K, Furuya T, Ishiyama S, Kim KJ, et al: Activated human T cells directly induce osteoclastogenesis from human monocytes: Possible role of T cells in bone destruction in rheumatoid arthritis patients. Arthritis Rheum 44: 1003-1012, 2001.

7. Harrison DE, Strong R, SharpZD, Nelson JF, Astle CM, Flurkey K, Nadon NL, Wilkinson JE, Frenkel K, Carter CS, et al: Rapamycin fed late in life extends lifespan in genetically heterogeneous mice. Nature 460: 392-395, 2009.

8. Guba M, von Breitenbuch P, Steinbauer M, Koehl G, Flegel S, Hornung M, Bruns CJ, Zuelke C, Farkas S, Anthuber M, et al: Rapamycin inhibits primary and metastatic tumor growth by antiangiogenesis: Involvement of vascular endothelial growth factor. Nat Med 8: 128-135, 2002.

9. Zheng N, Ding X and Jahan R: Low concentration of rapamycin inhibits hemangioma endothelial cell proliferation, migration, and vascular tumor formation in mice. Curr Ther Res Clin Exp 76: 99-103, 2014.

10. Cho HJ, Park J, Lee HW, Lee YS and Kim JB: Regulation of adipocyte differentiation and insulin action with rapamycin. Biochem Biophys Res Commun 321: 942-948, 2004.

11. Antonarakis ES, Carducci MA and Eisenberger MA: Nove targeted therapeutics for metastatic castration-resistant prostate cancer. Cancer Lett 291: 1-13, 2010.

12. Sarbassov DD, Ali SM, Sengupta S, Sheen JH, Hsu PP, Bagley AF, Markhard AL and Sabatini DM: Prolonged rapamycin treatmen inhibits mTORC2 assembly and Akt/PKB. Mol Cell 22: 159-168, 2006.

13. Yang Q and Guan KL: Expanding mTOR signaling. Cell Res 17: 666-681, 2007.

14. Lam J, Takeshita S, Barker JE, Kanagawa O, Ross FP and Teitelbaum SL: TNF-alpha induces osteoclastogenesis by direct stimulation of macrophages exposed to permissive levels of RANK ligand. J Clin Invest 106: 1481-1488, 2000.

15. Fielhaber JA, Carroll SF, Dydensborg AB, Shourian M, Triantafillopoulos A, Harel S, Hussain SN, Bouchard M, Qureshi ST and Kristof AS: Inhibition of mammalian target of rapamycin augments lipopolysaccharide-induced lung injury and apoptosis. J Immunol 188: 4535-4542, 2012.

16. Tulek B, Kiyan E, Toy H, Kiyici A, Narin C and Suerdem M: Anti-inflammatory and anti-fibrotic effects of sirolimus on bleomycin-induced pulmonary fibrosis in rats. Clin Invest Med 34: E341, 2011

17. Singha UK, Jiang Y, Yu S, Luo M, Lu Y, Zhang J and Xiao G: Rapamycin inhibits osteoblast proliferation and differentiation in MC3T3-E1 cells and primary mouse bone marrow stromal cells. J Cell Biochem 103: 434-446, 2008.

18. Shui C, Riggs BL and Khosla S: The immunosuppressant rapamycin, alone or with transforming growth factor-beta, enhances osteoclast differentiation of RAW264.7 monocyte-macrophage cells in the presence of RANK-ligand. Calcif Tissue Int 71: 437-446, 2002.

19. Phornphutkul C, Lee M, Voigt C, Wu KY, Ehrlich MG, Gruppuso PA and Chen Q: The effect of rapamycin on bone growth in rabbits. J Orthop Res 27: 1157-1161, 2009

20. Viñals F, López-Rovira T, Rosa JL and Ventura V: Inhibition of $\mathrm{PI} 3 \mathrm{~K} / \mathrm{p} 70 \mathrm{~S} 6 \mathrm{~K}$ and p38 MAPK cascades increases osteoblastic differentiation induced by BMP-2. FEBS Lett 510: 99-104, 2002

21. Lee KW, Yook JY, Son MY, Kim MJ, Koo DB, Han YM and Cho YS: Rapamycin promotes the osteoblastic differentiation of human embryonic stem cells by blocking the mTOR pathway and stimulating the BMP/Smad pathway. Stem Cells Dev 19: $557-568,2010$

22. Lian JB, Javed A, Zaidi SK, Lengner C, Montecino M van Wijnen AJ, Stein JL and Stein GS: Regulatory controls for osteoblast growth and differentiation: Role of Runx/Cbfa/AML factors. Crit Rev Eukaryot Gene Expr 14: 1-41, 2004.
23. Owen TA, Aronow M, Shalhoub V, Barone LM, Wilming L, Tassinari MS, Kennedy MB, Pockwinse S, Lian JB and Stein GS: Progressive development of the rat osteoblast phenotype in vitro: Reciprocal relationships in expression of genes associated with osteoblast proliferation and differentiation during formation of the bone extracellular matrix. J Cell Physiol 143: 420-430, 1990.

24. Stricker S, Fundele R, Vortkamp A and Mundlos S: Role of Runx genes in chondrocyte differentiation. Dev Biol 245: 95-108, 2002

25. Mohamadnia AR, Shahbazkia HR, Sharifi S and Shafael I: Bone-specific alkaline phosphatase as a good indicator of bone formation in sheepdogs. Comp Clin Path 16: 265-270, 2007.

26. Matsubara T, Kida K, Yamaguchi A, Hata K, Ichida F, Meguro H, Aburatani $\mathrm{H}$, Nishimura R and Yoneda T: BMP2 regulates Osterix through Msx2 and Runx2 during osteoblast differentiation. J Biol Chem 283: 29119-29125, 2008.

27. Nishio Y, Dong Y,Paris M, O'Keefe RJ, Schwarz EM and Drissi H: Runx2-mediated regulation of the zinc finger Osterix/Sp7 gene. Gene 372: 62-70, 2006.

28. Elsubeihi ES and Heersche JN: Quantitative assessment of post-extraction healing and alveolar ridge remodelling of the mandible in female rats. Arch Oral Biol 49: 401-412, 2004.

29. Sanchez CP and He YZ: Bone growth during rapamycin therapy in young rats. BMC Pediatr 9: 3, 2009.

30. Sun H, Kim JK, Mortensen R, Mutyaba LP, Hankenson KD and Krebsbach PH: Osteoblast-targeted suppression of PPAR $\gamma$ increases osteogenesis through activation of mTOR signaling. Stem Cells 31: 2183-2192, 2013.

31. Wang Y, Yi XD and Li CD: Suppression of mTOR signaling pathway promotes bone marrow mesenchymal stem cells differentiation into osteoblast in degenerative scoliosis: In vivo and in vitro. Mol Biol Rep 44: 129-137, 2017.

32. van der Bruggen T, Nijenhuis S, van Raaij E, Verhoef $\mathbf{J}$ and van Asbeck BS: Lipopolysaccharide-induced tumor necrosis factor alpha production by human monocytes involves the raf-1/MEK1-MEK2/ERK1-ERK2 pathway. Infect Immun 67: 3824-3829, 1999 .

33. Fisher CJ Jr, Agosti JM, Opal SM, Lowry SF, Balk RA, Sadoff JC, Abraham E, Schein RM and Benjamin E: Treatment of septic shock with the tumor necrosis factor receptor: $\mathrm{Fc}$ fusion protein. The soluble TNF receptor sepsis study group. N Engl J Med 334: $1697-1702,1996$

34. Glauser MP, Zanetti G, Baumgartner JD and Cohen J: Septic shock: Pathogenesis. Lancet 338: 732-736, 1991.

35. Sweet MJ and Hume DA: Endotoxin signal transduction in macrophages. J Leukoc Biol 60: 8-26, 1996.

36. Pacifici R, Brown C, Puscheck E, Friedrich E, Slatopolsky E, Maggio D, McCracken R and Avioli LV: Effect of surgical menopause and estrogen replacement on cytokine release from human blood mononuclear cells. Proc Natl Acad Sci USA 88: 5134-5138, 1991.

37. Manolagas SC, Bellido T and Jilka RL: New insights into the cellular, biochemical, and molecular basis of postmenopausal and senile osteoporosis: Roles of IL-6 and gp130. Int J Immunopharmacol 17: 109-116, 1995.

38. Hofbauer LC, Lacey DL, Dunstan CR, Spelsberg TC, Riggs BL and Khosla S: Interleukin-1beta and tumor necrosis factor-alpha, but not interleukin-6, stimulate osteoprotegerin ligand gene expression in human osteoblastic cells. Bone 25: 255-259, 1999.

39. Wei S, Kitaura H, Zhou P, Ross FP and Teitelbaum SL: IL-1 mediates TNF-induced osteoclastogenesis. J Clin Invest 115 282-290, 2005

40. Weitzmann MN, Roggia C, Toraldo G, Weitzmann L and Pacifici R: Increased production of IL-7 uncouples bone formation from bone resorption during estrogen deficiency. J Clin Invest 110: 1643-1650, 2002.

41. Krajewski AC, Biessei J, Kunze M, Maersch S, Perabo L and Noack MJ: Influence of lipopolysaccharide and interleukin-6 on RANKL and OPG expression and release in human periodontal ligament cells. APMIS 117: 746-754, 2009.

42. Powell N, Till S, Bungre J and Corrigan C: The immunomodulatory drugs cyclosporin A, mycophenolate mofetil, and sirolimus (rapamycin) inhibit allergen-induced proliferation and IL-5 production by PBMCs from atopic asthmatic patients. J Allergy Clin Immunol 108: 915-917, 2001.

43. Luo D, Ren H, Li T, Lian K and Lin D: Rapamycin reduces severity of senile osteoporosis by activating osteocyte autophagy. Osteoporos Int 27: 1093-1101, 2016. 